\title{
Successful Management of an Unusual Complication after Transjugular Intrahepatic Portosystemic Shunt Procedure
}

\author{
Nischal. G. Kundaragi ${ }^{1}$ Naresh Bhat ${ }^{2}$ Sudhir Kale ${ }^{3}$ \\ ${ }^{1}$ Department of Interventional Radiology, Aster CMI Hospital, \\ Bengaluru, Karnataka, India \\ 2Department of Gastroenterology and Liver Disease, Aster CMI \\ Hospital, Bengaluru, Karnataka, India \\ ${ }^{3}$ Department of Radiology, Aster CMI Hospital, Bengaluru, \\ Karnataka, India
}

J Clin Interv Radiol ISVIR 2020;4:60-63

Transjugular intrahepatic portosystemic shunt (TIPS) is a well-established treatment procedure in the management of sequelae of portal hypertension. Major complication rate of TIPS procedure is less than $5 \%$ and can have lethal consequences, if not recognized and treated early. ${ }^{1}$ Arterial compression and subsequent segmental hepatic ischemia/ infarction after TIPS procedure can be treated with antibiotic coverage and supportive care. ${ }^{2}$ We present an unusual early complication after a successful TIPS procedure. The patient presented with capsular/subcapsular active bleeding from infarcted liver associated with significant compression of the posterior branch of the right hepatic artery by the TIPS stent. This unique complication was recognized immediately and treated successfully by embolization and supportive care.

\section{Case Report}

A 73-year-old man with idiopathic cirrhosis presented with refractory ascites of 2 months duration. Patient has been on medical therapy for ascites for 1 year. He became refractory to medical management and required ascites fluid removal of $\sim 5$ to 6 L every week. A transjugular intrahepatic portosystemic shunt (TIPS) procedure was advised to reduce the portal hypertension and treat refractory ascites.

His laboratory findings were as follows: Hemoglobin (Hb) was $9.3 \mathrm{~g} / \mathrm{dL}$. Serum creatinine was $1.1 \mathrm{mg} / \mathrm{dL}$. Total bilirubin was $1.2 \mathrm{mg} / \mathrm{dL}$. Serum sodium was $134 \mathrm{meq} / \mathrm{L}$. Serum albumin was $3.7 \mathrm{~g} / \mathrm{dL}$. Platelet count was $114 \mathrm{k} / \mu \mathrm{L}$. The international normalized ratio (INR) was 1.5. The model for end-stage liver disease (MELD) score was 11 . No hepatic encephalopathy was observed.

A preprocedure contrast-enhanced computed tomography (CT) scan excluded hepatocellular carcinoma (HCC). An $8 \mathrm{~F}$ pigtail catheter was placed to drain ascites.

\begin{abstract}
Address for correspondence Nischal. G. Kundaragi, MDRD, DNB, FRCR, Department of Interventional Radiology, Aster CMI Hospital, Bengaluru 560092, Karnataka, India (e-mail: drngk@yahoo.com).
\end{abstract}

Transjugular intrahepatic portosystemic shunt procedure was performed through a right transjugular vein approach. Rösch-Uchida set (Cook Medical) was used for puncturing the portal vein (PV). The right main PV was punctured from the right hepatic vein. Ultrasound guidance was used for puncture and the portal vein was accessed in 3 attempts. No arterial puncture was done during the procedure. Serial balloon dilatation of the tract was performed. Prestenting, por-

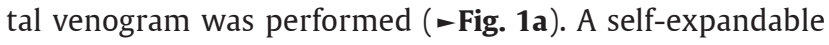
Niti-S (Taewoong) TIPS stent of size $10 \times 90 \mathrm{~mm}(70 \mathrm{~mm}$ covered intrahepatic portion and $20 \mathrm{~mm}$ uncovered PV portion) was deployed. Poststenting, the stent was dilated to $10 \mathrm{~mm}$. A portal venogram revealed widely patent portosystemic shunt between the right PV and right hepatic vein ( - Fig. 1b). Poststenting, pressure gradient reduced from 26 to $8 \mathrm{~mm} \mathrm{Hg}$. Unfractionated heparin (2500 IU) was used for anticoagulation. Spectral Doppler ultrasound done immediately after the procedure showed normal velocity (average $180 \mathrm{~cm} / \mathrm{s}$ ) in the portosystemic shunt and no obvious hematoma or perihepatic free fluid or collection. No change in color was observed in ascites drain catheter. Ascites drain color was clear till 4 hours after the procedure. After 4 hours, blood-stained ascites drain was seen. Tachycardia was observed with 100 to $110 \mathrm{bpm}$; however, no significant decrease in $\mathrm{Hb}$ was noted. Further planned anticoagulation was withheld. A 6-hourly monitored $\mathrm{Hb}$ showed a decrease of 0.5 to $0.7 \mathrm{~g} / \mathrm{dL}$ over 12 hours $(8.1 \mathrm{~g} / \mathrm{dL}$ after $12 \mathrm{hr}$ ) and the patient developed persistent tachycardia (110 bpm). Hemoperitoneum was observed on ultrasound examination. Liver function tests and Contrast Enhanced CT (CECT) scan were performed. Serum total bilirubin increased to $1.48 \mathrm{mg} / \mathrm{dL}$, aspartate aminotransferase (AST) $1123 \mathrm{U} / \mathrm{L}$, alanine aminotransferase (ALT) $930 \mathrm{U} / \mathrm{L}$, serum alkaline phosphatase (ALP) $450 \mathrm{U} / \mathrm{L}$, serum sodium $130 \mathrm{meq} / \mathrm{L}$, serum albumin $3.5 \mathrm{~g} / \mathrm{dL}$, platelet count $103 \mathrm{k} / \mu \mathrm{L}$, and the INR 2.4. CECT revealed hemoperitoneum
License terms

$10.1055 / \mathrm{s}-0039-3401307$

ISSN 2457-0214. 
and wedge-shaped infarcted area in segment 6, and part of segments 5 and 7 of the right lobe of the liver. Arterial phase study revealed focal linear abnormal contrast blush in the subcapsular region of segment 6 with attenuated posterior branches of right hepatic artery (-Fig. 2a-c). Two units of whole blood were transfused. Immediate angiography and embolization was performed. Digital angiogram revealed TIPS stent causing significant nonocclusive compression of posterior branch of right hepatic artery. Super selective digital subtraction angiogram (DSA) revealed abnormal parenchymal blush and focal arterial ectasia matching CECT scan. Embolization was performed initially with gel-foam and later with $3 \times 3 \mathrm{~mm}$ 018' microcoil (Cook Medical) (-Fig. 3a-c). Postembolization, Hb and heart rate (84 bpm) were stabilized. Before discharge, on 5th postembolization day, Hb was $12.3 \mathrm{~g} / \mathrm{dL}$, AST was $126 \mathrm{U} / \mathrm{L}$, and ALT was 206 U/L. Ascites drain tube was removed after draining $3 \mathrm{~L}$ of blood-stained fluid. Post discharge, at 6 weeks follow-up,

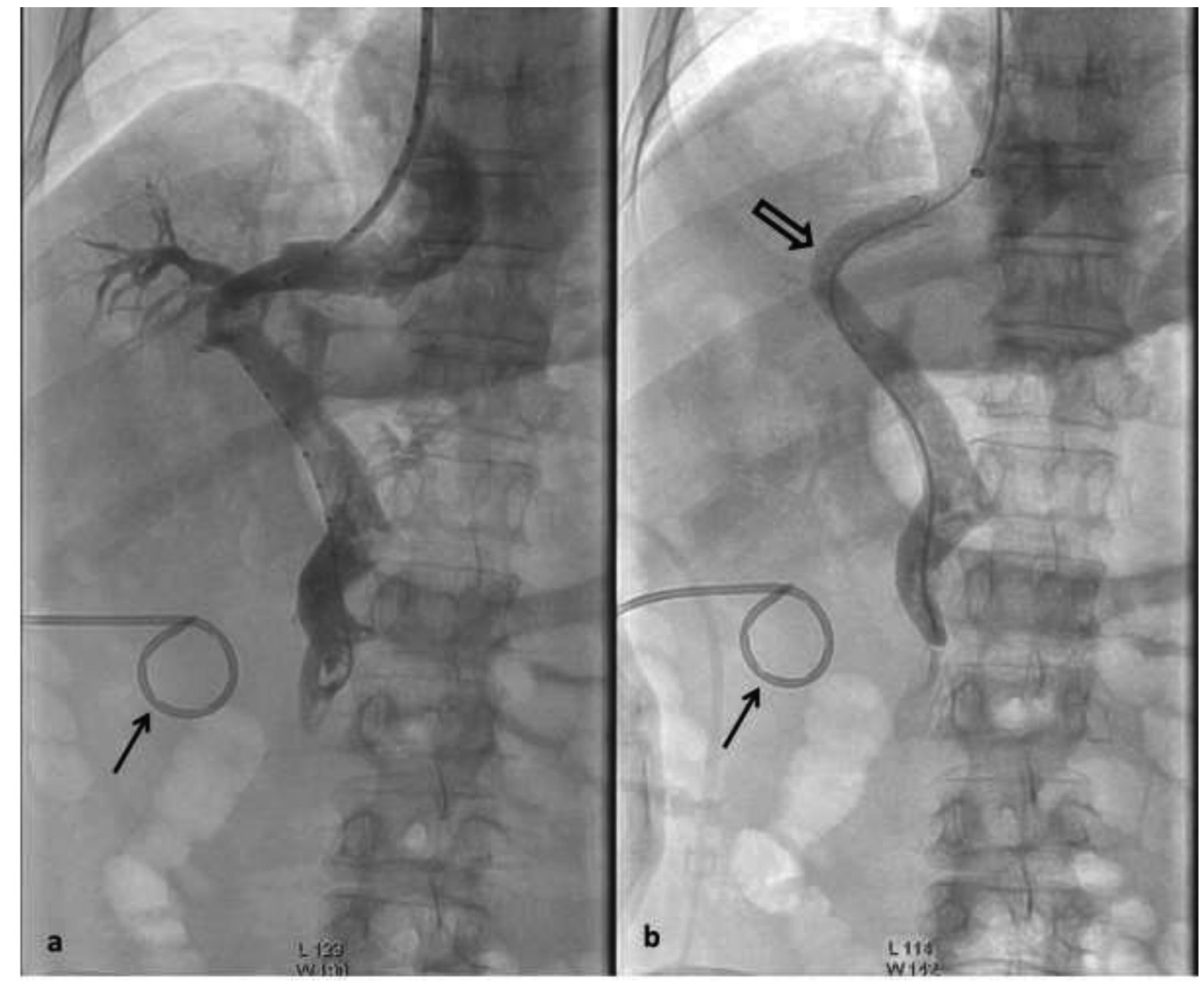

Fig. 1 A 73-year-old man, known case of cirrhosis, presented with refractory ascites. (a) Prestenting, digital portal venogram done through marker pigtail catheter and long 10F sheath showing portal vein (PV) and right hepatic vein. (b) Poststenting, digital venogram showing patent stent with (open black arrow) with opacified distal superior mesenteric vein (SMV) and portal vein. Pigtail catheter (8F) placed for ascites drainage (black arrows).

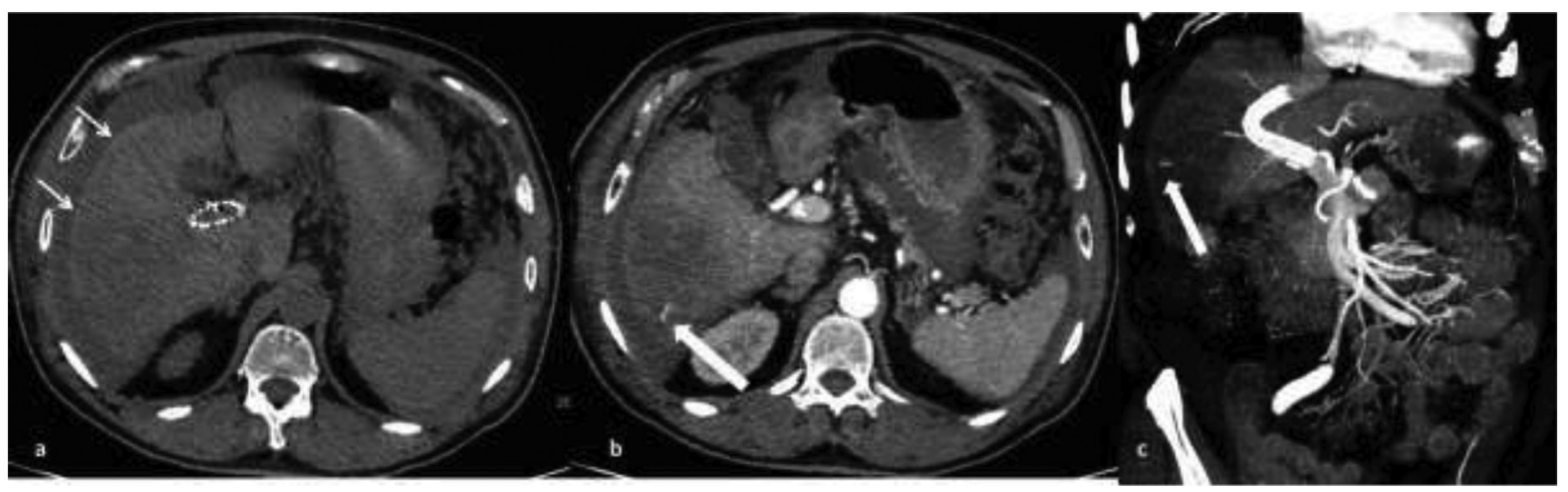

Fig. 2 A 73-year-old man, known case of cirrhosis, presented with refractory ascites. (a) NECT scan showing hemoperitoneum (white arrows). (b, c) CECT scan arterial phase, axial and coronal images showing abnormal contrast blush (thick white arrow) in subcapsular area of segment 6 of right lobe of liver. Wedge-shaped hypoenhancing area seen in visualized right lobe of liver around the abnormal arterial blush. 


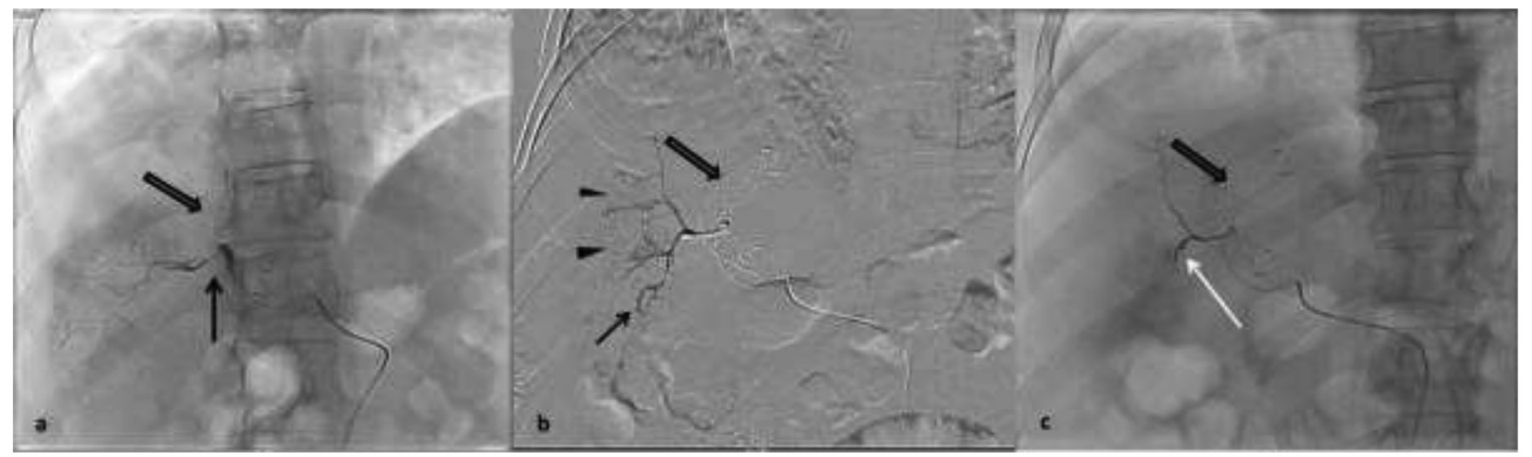

Fig. 3 A 73-year-old man, known case of cirrhosis, presented with refractory ascites. (a) Digital angiogram of posterior sectoral branch of right hepatic artery (RHA) showing significant compression (black arrow) by TIPS stent. No dissection or thrombotic segments were seen. (b) Superselctive digital subtraction angiogram of posterior sectoral branch of RHA showing abnormal contrast blush in segment 6 artery and abnormal blush in adjacent parenchyma (arrow heads). (c) Digital angiogram after successful gelfoam and coil (white arrow) embolization. TIPS stent (black open arrows).

no significant ascites was observed. Total serum bilirubin was $2.09 \mathrm{mg} / \mathrm{dL}$ (direct was $0.7 \mathrm{mg} / \mathrm{dL}$ and indirect was $1.39 \mathrm{mg} / \mathrm{dL}$ ), AST was $125 \mathrm{U} / \mathrm{L}$, ALT was $66 \mathrm{U} / \mathrm{L}$, and ALP was $105 \mathrm{U} / \mathrm{L}$. The patient had grade I hepatic encephalopathy with no recurrence of ascites at 5 months follow-up period.

\section{Discussion}

Preprocedural assessment of hepatic function, coagulation, and imaging (ultrasonography, CT scan, or magnetic resonance imaging) are important before TIPS procedure to reduce postprocedural complications. ${ }^{2}$ Model for end-stage liver disease (MELD), The Acute Physiology and Chronic Health Evaluation (APACHE II), and ChildPugh scores are used to predict survival following TIPS. However, three-month and one-year survival were better predicted by MELD score compared with the other two scoring systems. ${ }^{3}$

Major complication rate of TIPS procedure is less than 5\%. ${ }^{1}$ Reported possible complications include TIPS dysfunction (thrombosis, occlusion, or stenosis) in 10 to $15 \%$, transcapsular puncture in $33 \%$, hemobilia in $~ 5 \%$, sepsis in 2 to $10 \%$, intraperitoneal bleed in 1 to $2 \%$, hepatic infarction in $~ 1 \%$, hemolysis in 10 to $15 \%$, encephalopathy in 5 to $44 \%$, and stent malposition in 10 to $20 \%$ of cases. Other rare complications include acute hepatic failure, carotid artery injuries, right atrial perforation, portal vein perforation, fistulae formation, hernia incarceration, infection of TIPS stent, and radiation injuries..$^{1-3}$

Serious arterial complications like hepatic artery injury, liver laceration, intrahepatic hematomas, and acute intra-abdominal hemorrhage can occasionally occur in the early postprocedural period up to 2 weeks following TIPS placement in a patient receiving low molecular weight heparin or warfarin. ${ }^{2}$

Hepatic artery injury during TIPS procedure is seen in 1 to $6 \%$ cases. It is not uncommon to puncture the intraparenchymal hepatic artery branches during a TIPS procedure. However, precautions must be taken by the operator to identify the hepatic arterial puncture, not to advance the catheter, to withdraw the catheter if advanced, and to embolize the tract whenever required to avoid lethal complications. ${ }^{1,4,5}$

In patients with suspected postprocedural bleeding complication, CT scan should be performed to identify the source of bleeding. Anticoagulant medications should be stopped and reversed if possible. If an arterial source is identified, hepatic arteriography and embolization should be performed. $^{2}$

Segmental liver ischemia and infarction after TIPS procedure had been reported with polytetrafluoroethylene (PTFE)-covered stents.6-8 Segmental infarction was also reported in post liver transplant case with poor outcome. ${ }^{9}$ Transient segmental liver ischemia can happen due to hepatic venous outflow obstruction by PTFE stents, which usually resolves with the development of collaterals allowing the right hepatic vein to drain into the left and middle hepatic veins. ${ }^{8}$ Hepatic perfusion after TIPS depends on the arterial buffer reserve. ${ }^{10}$ Stent compression of the hepatic artery can also cause hepatic ischemia or infarction. ${ }^{2,4,11}$ Ischemia may turn into infarction if portal and hepatic venous flow obstruction are associated with arterial pathology such as compression by TIPS stent or postoperative stenosis. ${ }^{2,49,11}$ Similarly, in this unique case, post TIPS CT scan done to identify the source of bleeding revealed significant stenosis of posterior sectoral branch of right hepatic artery by TIPS stent

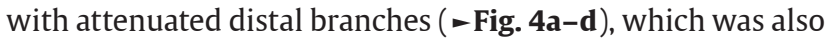
confirmed during digital angiogram and embolization.

Arterial compression by the TIPS stent with subsequent segmental hepatic infarction can be treated with antibiotic coverage and supportive care. ${ }^{2}$ However, in presented case, CT scan revealed bleeding from infarcted areas with subcapsular linear arterial abnormality and hemoperitoneum. This was successfully treated by gel-foam and coil embolization. Post embolization, patient was under antibiotic coverage for specified period and received supportive care under medical gastroenterology team.

In view of significant arterial stenosis by TIPS stent, anticoagulation (low molecular weight heparin) was started after 2 weeks. In our institution, we routinely use anticoagulation after TIPS procedure for 6 weeks. In these type 


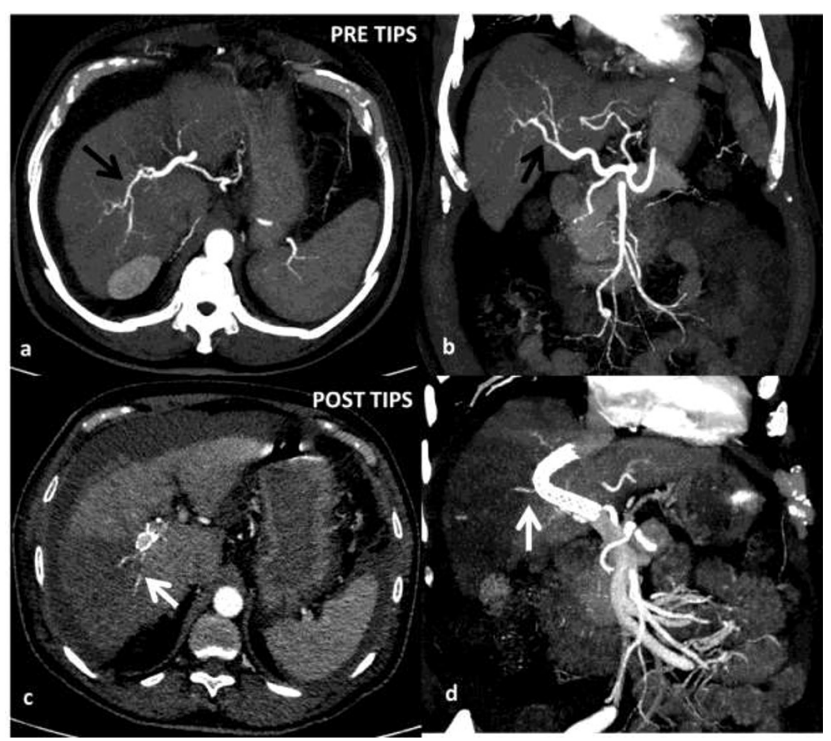

Fig. 4 A 73-year-old man, known case of cirrhosis, presented with refractory ascites. Pre- and post-TIPS comparison of CECT scan arterial phase, axial and coronal images. (a, b) Pre-TIPS. MIP images showing normal anterior and posterior sectoral branches of RHA. (c, d) Post-TIPS. CT scan images showing attenuated posterior sectoral branches of RHA.

of cases, anticoagulation acts as a double-edged sword. If arterial stenosis is significant, anticoagulation will help; however, conversely it might lead to parenchymal bleeding in infarcted liver.

\section{Conclusion}

In conclusion, rare early complication like significant arterial stenosis, segmental infarction, and associated bleeding from infarcted area can happen after successful TIPS procedure. Early detection of such complications and treatment will prevent further liver damage and its sequelae.

\section{Conflict of Interest}

None.

\section{References}

1 Ripamonti R, Ferral H, Alonzo M, Patel NH. Transjugular intrahepatic portosystemic shunt-related complications and practical solutions. Semin Intervent Radiol 2006;23(2):165-176

2 Suhocki PV, Lungren MP, Kapoor B, Kim CY. Transjugular intrahepatic portosystemic shunt complications: prevention and management. Semin Intervent Radiol 2015;32(2):123-132

3 Boyer TD, Haskal ZJ. AASLD practice guideline update. The role of transjugular intrahepatic portosystemic shunt (TIPS) in the management of portal hypertension: update 2009. Hepatology 2010;57:1-16

4 Gaba RC, Khiatani VL, Knuttinen MG, et al. Comprehensive review of TIPS technical complications and how to avoid them. AJR Am J Roentgenol 2011;196(3):675-685

5 Haskal ZJ, Cope C, Shlansky-Goldberg RD, et al. Transjugular intrahepatic portosystemic shunt-related arterial injuries: prospective comparison of large- and small-gauge needle systems. J Vasc Interv Radiol 1995;6(6):911-915

6 Bureau C, Otal P, Chabbert V, Péron JM, Rousseau H, Vinel JP. Segmental liver ischemia after TIPS procedure using a new PTFE-covered stent. Hepatology 2002;36(6):1554

7 Lopera JE, Katabathina V, Bosworth B, et al. Segmental liver ischemia/infarction after elective transjugular intrahepatic portosystemic shunt creation: clinical outcomes in 10 patients. J Vasc Interv Radiol 2015;26(6):835-841

8 Ferguson JW, Tripathi D, Redhead DN, Ireland H, Hayes PC. Transient segmental liver ischaemia after polytetrafluoroethylene transjugular intrahepatic portosystemic stent-shunt procedure. J Hepatol 2005;42(1):145

9 Wang MY, Potosky DR, Khurana S. Liver infarction after transjugular intrahepatic portosystemic shunt in a liver transplant recipient. Hepatology 2011;54(5):1887-1888

10 Zipprich A. Hemodynamics in the isolated cirrhotic liver. J Clin Gastroenterol 2007;41(Suppl 3):S254-S258

11 Vizzutti F, Arena U, Rega L, et al. Liver failure complicating segmental hepatic ischaemia induced by a PTFE-coated TIPS stent. Gut 2009;58(4):582-584 